\title{
Oral 4-aminosalicylic acid versus 5-aminosalicylic acid slow release tablets. Double blind, controlled pilot study in the maintenance treatment of Crohn's ileocolitis
}

\author{
S Schreiber, S Howaldt, A Raedler
}

\begin{abstract}
4-Aminosalicylic acid (4-ASA) has been suggested as an effective treatment for both active and quiescent ulcerative colitis. 5-Aminosalicylic acid (5-ASA) is well accepted for the maintenance treatment of inactive ulcerative colitis. Moreover, recent studies suggest that 5-ASA may also be effective in maintaining remission in Crohn's colitis. As treatment with 4-ASA may result in less side effects, the efficacy of a one year's maintenance treatment with oral 4-ASA $(1.5 \mathrm{~g} / \mathrm{d}$, slow release tablets, $n=19)$ and oral 5 -ASA $(1.5 \mathrm{~g} / \mathrm{d}$, slow release tablets, $n=21$ ) was compared in a double blind, randomised trial in patients with quiescent Crohn's ileocolitis. Patients with ileocolonic or colonic involvement were enrolled if in stable remission for more than two months but less than one year. Baseline demography and clinical severity were similar in both groups. Total colonoscopy and ileoscopy were performed at enrolment and at the end of the study. After one year seven of 19 patients receiving 4-ASA $(36 \%)$ and 8 of 21 receiving 5-ASA $(38 \%)$ had developed a clinical relapse, as defined by a rise in the Crohn's disease activity index (CDAI) of more than 100 points to values higher than 150. The relapse rates between the 4-ASA and the 5-ASA groups were not statistically different although no comparison with the spontaneous relapse rate in a placebo group could be made. Clinical relapse was accompanied by a statistically significant rise in serum concentrations of soluble interleukin 2 receptor and by an increased percentage of activated peripheral blood $T$ cells. There were no statistical differences between the 4-ASA and the 5-ASA groups regarding the height of rise in CDAI or of soluble interleukin 2 receptor concentrations during relapse, thus showing a similar severity of relapsed disease activity. In conclusion, 4-ASA may be as effective as 5-ASA in the maintenance treatment of quiescent Crohn's disease and there were no differences in the severity of relapse between both treatment groups.

(Gut 1994; 35: 1081-1085)
\end{abstract}

4-Aminosalicylic acid (4-ASA), also named para-aminosalicylic acid, is an agent, well known as a comparatively safe drug from its use in high doses for the treatment of tuberculosis over many years. It is an isomer of 5-aminosalicylic acid (5-ASA), which is the active component of the salazosulphapyridine molecule in the treatment of inflammatory bowel disease (IBD). 5-ASA compounds are presently used for the treatment of both moderately active Crohn's disease $^{1-4}$ and active ulcerative colitis. ${ }^{5-7}$ It has been shown that 5-ASA is also effective in maintaining remission in patients with ulcerative colitis, ${ }^{78}$ whereas its value for maintenance treatment of quiescent Crohn's disease is disputed: some authors see no significant difference in the rate of relapse between 5-ASA and placebo, ${ }^{910}$ whereas in other trials 5-ASA may be effective in maintaining remission. ${ }^{1-13}$ The efficacy of 5-ASA, however, in the maintenance of remission in Crohn's disease may be higher in ileal disease ${ }^{13}$ or when given during the first three months after remission was achieved. ${ }^{12}$

In comparison with 5-ASA, the sodium salt of 4-ASA is a more stable compound. ${ }^{14} 15$ Animal models led to the conclusion that 4-ASA may have anti-inflammatory properties comparable with those of 5-ASA. ${ }^{14}$ Numerous clinical studies have shown that 4-ASA is highly effective in the topical treatment of active ulcerative proctitis or active left sided ulcerative colitis. ${ }^{20}$ Moreover, 4-ASA slow release tablets were shown to be effective in active ulcerative colitis. ${ }^{21}$ We now report the results of a one year, double blind, randomised, and prospective pilot trial, in which enterically coated 4-ASA was compared with 5-ASA at $0.5 \mathrm{~g}$ thrice daily in the maintenance treatment of patients with inactive Crohn's colitis and ileocolitis. A placebo group could not be included for ethical reasons because 5-ASA is widely used for the treatment of inactive Crohn's disease in Germany.

\section{Methods}

STUDY CRITERIA

The following criteria were required of all patients to be included in the study: Crohn's colitis or ileocolitis in a stable remission for 
more than two months with a Crohn's disease activity index (CDAI) $<150$ points. When patients were randomised, they were comparable with respect to age, sex, duration of disease, disease activity, symptoms, and adjunctive treatment as far as permitted by the study protocol. Patients were required not to have taken any active drugs for the treatment of IBD such as 5-aminosalicylic acid (5-ASA), salazosulphapyridine or steroids one week before entry to the study. Patients receiving methotrexate, azathioprine or 6-mercaptopurine were excluded. The extent and localisation of disease had to be verified from endoscopy during the last active phase. Patients with less than $30 \mathrm{~cm}$ of diseased bowel were excluded from the study as were those patients in a stable remission for more than one year or with bowel resections $>50 \mathrm{~cm}$. Further exclusion criteria were recorded as previous non-responsiveness to 5-ASA, hypersensitivity to 5-ASA compounds, and pregnancy or lactation, or both. Patients with known active ulcer disease (stomach or duodenal, or both) as well as patients with known disease manifestations in the upper gastrointestinal tract, with fistulas or with stenoses were also excluded. A medical approved plan of contraception was required from all menstruating women. If it was felt that the medical problems the patient developed during the study resulted from the progressive formation of stenoses without signs of active inflammation, the patient was withdrawn from the study. Defined end points were the end of the one year study period or a relapse in disease activity as defined by a rise of the CDAI of more than 100 points to a resulting value $>150$. The trial was granted prior approval by the institutional review boards of the University of Hamburg and all patients signed an informed consent. A placebo group was not included because of ethical reasons (benefit by 5-ASA, which is widely used for maintenance of remission in Germany).

\section{PATIENTS}

Sixty patients seen at the inflammatory bowel disease clinics of the University of Hamburg were enrolled into the trial and were prospectively randomised on 5-ASA and 4-ASA. Groups were comparable in disease manifestation, length of present remission, and previous treatment (Table I). Study patients were drawn from the outpatient population with

TABLE I Disease characteristics of the study population

\begin{tabular}{lccc}
\hline & $4-A S A$ group & 5-ASA group & $\begin{array}{c}\text { Total outpatient } \\
\text { population (\%) }\end{array}$ \\
\hline Disease in upper gastrointestinal tract & 0 & 0 & 6 \\
lleal disease & 93 & 97 & 87 \\
Crohn's colitis & 100 & 100 & 69 \\
Rectum affected & 47 & 40 & 21 \\
Previous resections & 37 & 27 & 39 \\
Less than three previous relapses & 47 & 57 & 52 \\
Average duration of disease (years) & $3 \cdot 3$ & $3 \cdot 7$ & $5 \cdot 2$ \\
Average duration of remission (months) & $4 \cdot 3$ & $5 \cdot 7$ & NA
\end{tabular}

Patients in the 4-ASA and the 5-ASA groups were comparable with regard to the disease characteristics. No differences in extent and history of disease were seen. Baseline demographics as well as previous treatment were identical between the 4-ASA and the 5-ASA groups (data not shown). NA = not assessed.
Crohn's disease at the University of Hamburg (237 patients). This population had disease manifestation in the upper gastrointestinal tract in $6 \%$ of cases, in the terminal ileum in $87 \%$, in the colon in $69 \%$, and the rectum in $21 \%$. Eight patients in the 5-ASA group and 11 patients in the 4-ASA group had had previous colonic or ileocolonic resections. In $96 \%$ of patients with Crohn's colitis involvement of the ileum was seen. Of all patients screened with ileocolonic disease $39 \%$ fulfilled the inclusion criteria. One patient was excluded because of hypersensitivity to 5-ASA, no patient because of a reported unresponsiveness to 5-ASA. Forty patients (19 receiving 4-ASA and 21 receiving 5-ASA) completed the study, three patients developed progressive stenoses, the remaining 17 patients were withdrawn from the trial because of unreliability in keeping their appointments. None of the patients withdrawn developed a clinical relapse within four weeks after the exclusion from the study.

PROTOCOL

At the first visit, patient history, physical examination, colonoscopy, stool examination for ova, parasites, and enteric pathogens were carried out, blood was taken for the assessment of electrolytes, differential blood count, sequential multiple analysis (SMA 12), determination of activated $\mathrm{T}$ cells, and serum soluble interleukin 2 receptor concentration. Office visits were repeated at monthy intervals, the patients kept daily diaries, in which they recorded the number of bowel movements and scores for blood, mucus, urgency, abdominal pain, and the appearance of extraintestinal manifestations. Each office visit included a physical examination, serum, electrolytes, differential blood count, and SMA 12. Every three months assessment of soluble interleukin 2 receptor and activated $T$ cells were repeated. Patients were randomly assigned to take either $500 \mathrm{mg}$ 4-ASA thrice daily or $500 \mathrm{mg} 5$-ASA thrice daily. The 4-ASA tablets were provided by Reed and Carnrick Inc/Block Drug Company Syngen GmbH (Piscataway, NJ, Ratingen, Germany), 5-ASA tablets were of the Claversal brand by SmithKline Beecham (Philadelphia, PA, Munich, Germany). Tablets were coated with Eudragit $L$ designed to dissolve at $\mathrm{pH} 6 \cdot 4$. No other drugs for the treatment of IBD were permitted during the study.

Colonoscopy and ileoscopy were performed at enrolment and at the study end point by an endoscopist not aware of the medication. Table II shows the results as classified by an endoscopy score.

ASSESSMENT OF ACTIVATED T CELLS AND SERUM SOLUBLE INTERLEUKIN 2 RECEPTOR CONCENTRATIONS

Peripheral blood mononuclear cells were prepared by density gradient centrifugation as described previously. ${ }^{22} 23$ The state of peripheral blood $\mathrm{T}$ cell activation was deter- 
TABLE II Endoscopy score used to assess disease activity

\begin{tabular}{ll}
\hline Score & Criteria \\
\hline 0 & Vascular pattern present, normal light reflex \\
1 & No vessels, granulations \\
2 & Contact bleeding, erythema \\
3 & Fibrinous exsudate, small ulcers $(<5 \mathrm{~mm}$, \\
& $<10 / 10 \mathrm{~cm})$ \\
4 & Spontaneous bleeding, large ulcers $(>5 \mathrm{~mm}$, \\
& $>10 \mathrm{~mm} / 10 \mathrm{~cm})$
\end{tabular}

Endoscopy scores were assessed by an endoscopist not participating in the study in a double blinded fashion. All endoscopies were carried out by the same examiner.

mined by the expression of transferrin receptors on their plasma membrane. ${ }^{22} 24$ In brief, mononuclear cells were labelled with fluorescent antibodies, specifically directed against CD3 ( $\mathrm{T}$ cells, fluorescein-isothiocyanate conjugated, Becton-Dickinson, Mountain View, CA) and the transferrin receptor (phycoerythrin conjugated, BectonDickinson). Cells were then analysed by two colour flow cytometry (Facsscan, BectonDickinson). ${ }^{22}$ Serum soluble interleukin 2 receptor concentrations were assessed by sandwich enzyme linked immunosorbent assay (ELISA) (T Cell Sciences, Cambridge, $\mathrm{MA}) .{ }^{23}$

\section{Results}

Of 60 patients, 21 receiving 5-ASA and 19 receiving 4-ASA completed the study by reaching a defined study end point (clinical relapse or one year of successful maintenance of remission). The remaining 20 patients were dropped from the trial because of progressive stenoses without significant inflammation (3) or non-compliance (17). Careful analysis showed that none of them left the study because of an inflammatory relapse. Neither the per protocol ( 40 patients) or the intention to treat analysis (60 patients) showed any statistical differences between the 4-ASA and the 5-ASA group (Fig 1). Of the patients who completed the protocol (40) the following cumulative relapse rates were calculated: after

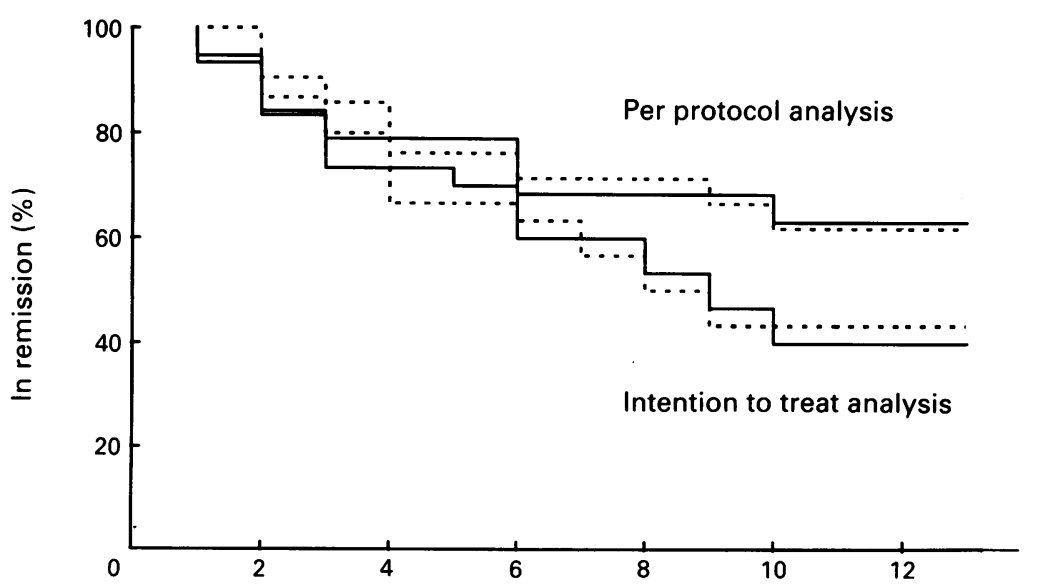

Trial duration (m)

Figure 1: Life table analysis of relapses under 4-ASA or 5-ASA, respectively. The upper two graphs represent the per protocol analysis of those patients that completed the trial two graphs represent the per protocol analysis of those patients that completed the trial
(19 receiving 4- $A S A, 21$ receiving $5-A S A)$, whereas the lower graphs show the intention to treat analysis of all patients enrolled into the trial ( 30 patients each group). Patients receiving 4-ASA are shown by the solid lines, patients under 5-ASA are shown by the dotted lines. In both data analyses, no statistically significant difference was seen between the groups. three months $21 \%(4 / 19)$ patients in the 4-ASA group and $14 \%(3 / 21)$ patients in the 5-ASA group had suffered from a relapse, at six months $32 \%$ of the 4-ASA patients and $29 \%$ in the 5 -ASA group (six patients in each group) had relapsed, and at 12 months in $36 \%$ (7/19) patients receiving 4-ASA and in 38\% $(8 / 21)$ patients receiving 5-ASA a relapse was seen. No statistically significant differences regarding disease history or localisation were seen between patients representing treatment failures and those remaining successfully in remission (data not shown).

The drop out patients as well as those excluded, who developed progressive stenoses, could also represent a subgroup of treatment failures (Fig 1). We therefore attempted to analyse the course of these patients. Eleven of 20 patients who had not completed the trial for the reasons stated above had been randomised to 4-ASA and 9 of 20 patients received 5-ASA. Of the three patients who had developed progressive stenoses, two received 4-ASA, one was in the 5-ASA group. All three patients developing stenoses had colonoscopy and no significant mucosal inflammation was seen. Moreover, we did not see a rise in the CDAI of more than 50 points and the laboratory constellation (C) reactive protein, erythrocyte sedimentation rate, soluble interleukin 2 receptor) did not suggest an acute inflammatory relapse. All 17 patients who had missed their appointments, have been contacted. None of them had left the study because of a rise of activity within four weeks of leaving the trial, which would have qualified them as a treatment failure.

We were interested to find out if the clinical severity of relapse was different between those patients treated with 4-ASA and those receiving 5-ASA. We therefore assessed the average rise in CDAI scores before and after relapse in a per protocol analysis of both groups. The CDAI values mean (SEM) of the relapsing patients treated with 4-ASA increased by 132 (36) points whereas the 5-ASA group showed an average rise of 147 (40) points. Moreover, average endoscopic scores mean (SEM) in those patients relapsing were not different between both treatment groups (3.1 (1.2) for 4-ASA and 3.5 (0.9) for 5-ASA treated patients). We also checked if disease activities were different between patients successfully maintaining remission receiving 4-ASA in comparison with those receiving 5-ASA. Again, no difference in CDAI scores was seen between both groups of quiescent patients (83.3 (29) and 60.9 (23.4) points, respectively).

Immunological parameters including serum soluble interleukin 2 receptor concentrations and activated peripheral blood $T$ cells can be used to monitor disease activity in IBD. ${ }^{22-24}$ 25-27 A difference in the immunological degree of activation would be indicative for differences in disease activity during relapse in either of the treatment groups. A clinical relapse was accompanied by an increase in serum soluble interleukin 2 receptor concentrations in both groups (Fig 2). Both the rise of 


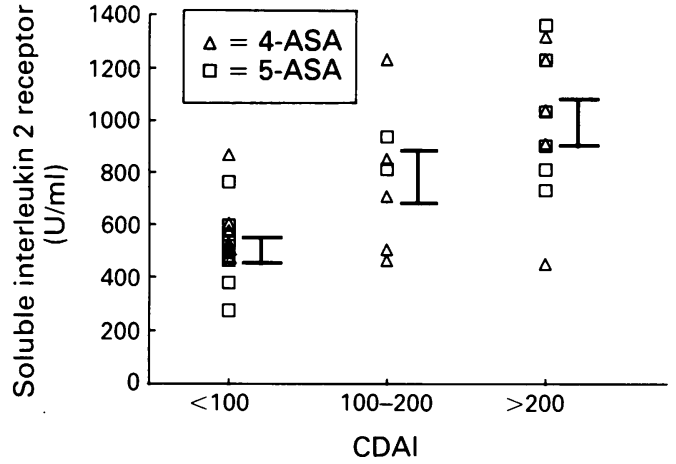

Figure 2: Correlation of serum soluble interleukin 2 receptor concentrations (sIL2R) to clinical disease activity (CDAI). Patients were grouped into those with a CDAI $<100$ $(n=21$, sIL2R: 527 (28.3) (mean (SEM)), from 100 to 200 ( $n=7$, sIL2R: 787 (92.2), $p=0.002$ compared with the patients with a $C D A I<100)$ and those with a $C D A I$ $>200(n=11$, sIL2R: 1003 (79.9), $p<0.001$ compared with the patients with a $C D A I<100)$. The error bars show mean (SEM).

serum soluble interleukin 2 receptor concentrations (mean (SEM)) during relapse (286 (48) U/ml (4-ASA) and $242(54) \mathrm{U} / \mathrm{ml}$ 5-ASA)) and the concentrations of activated T cells $10 \cdot 1(3 \cdot 4) \%(4-A S A)$ and $13 \cdot 5(4) \%$ (5-ASA) were similar in both groups. The increase in soluble interleukin 2 receptor concentrations during relapse was statistically significant $(p<0.01)$, when compared with interleukin 2 receptor values of clinically quiescent patients reaching the one year study end point (Fig 2).

Moreover, serum concentrations of soluble interleukin 2 receptors correlated in this study with clinical disease activity (Fig 2). When patients were grouped in those with a CDAI <100, with a CDAI from 100 to 200 , and those with a CDAI $>200$ the average serum soluble interleukin 2 receptor concentrations were significantly different (Fig 2). A linear regression analysis calculated a correlation coefficient of 0.732 between the soluble interleukin 2 receptor measurements shown in Fig 2 and the corresponding CDAI values.

\section{Discussion}

This pilot trial shows that oral 4-ASA may be as equally effective as 5-ASA in maintaining remission in quiescent Crohn's disease. Relapse rates were similar between patients treated with 4-ASA and those treated with 5-ASA. This trial therefore shows equivalent efficacy of both drugs in remission maintenance. The question whether 5-ASA is effective in maintaining remission of quiescent Crohn's disease is disputed: some studies found no efficacy $v$ placebo ${ }^{9} 10$ whereas others have seen a value of 5-ASA in the maintenance treatment of Crohn's disease ${ }^{1-13}$ particularly in patients with ileitis ${ }^{13}$ and in the first three months of remission. ${ }^{12}$ Unfortunately, a placebo group could not be included so that the data presented do not help to answer the question whether 5-ASA is effective in maintaining remission of Crohn's colitis or ileocolitis.
It is possible that the severity of relapse would be different under treatment with 4-ASA in comparison with 5-ASA. Neither by clinical (CDAI, endoscopic score) or by immunological parameters (serum soluble interleukin 2 receptor, activated $\mathrm{T}$ cells), however, could any difference in disease activity during relapse be found. Moreover, clinical disease activities (CDAI) of those patients successfully maintained in remission were similar whether treated by 4-ASA or by 5-ASA.

A correlation of serum soluble interleukin 2 receptor concentrations to disease activity has been suggested by a number of studies. ${ }^{2325-27}$ The use of serum soluble interleukin 2 receptor concentrations in this study underlines the concept that assessment of this molecule from frozen serum samples may be used to objectively determine and monitor disease activity in clinical trials.

4-Aminosalicylic acid differs from 5-ASA in that it is more stable than 5-ASA, which is substituted in meta position and which therefore brakes down more rapidly in a water solution. ${ }^{1415}$ In contrast with 5-ASA no nephrotoxicity related to 4-ASA has been reported. The only other study that compared these two compounds examined the response to topical treatment in left sided colitis and showed that 4-ASA and 5-ASA were equally effective. ${ }^{19}$

The anti-inflammatory mechanism by which the aminosalicylates exert their therapeutic action is unknown. 5-Aminosalicylic acid is a potent inhibitor of arachidonic acid metabolism, decreasing the synthesis of both leukotrienes and prostaglandins. ${ }^{28-30}$ Moreover, 5-ASA is a potent scavenger of free radicals. ${ }^{31-33}$ In contrast, 4-ASA does not seem to have an inhibitory effect of the lipoxygenation of arachidonic acid and is ineffective as a radical scavenger. ${ }^{34}$ Both drugs, however, inhibit in vitro activation of $\mathrm{T}$ and $\mathrm{B}$ lymphocytes by pokeweed mitogen in a dose dependent manner. ${ }^{22}{ }^{35}$ Animal models suggest that 4-ASA may be equally effective as 5-ASA with regard to its in vivo anti-inflammatory properties. ${ }^{1436}$ These findings suggest that the use of either drug in IBD may decrease the heightened state of lamina propria lymphocyte activation as a part of their therapeutic action. Moreover, mechanisms not affecting arachidonic acid metabolism and superoxide release may contribute to the therapeutic potential of both drugs in IBD.

4-Aminosalicylic acid, which has been previously named para-aminosalicylic acid was used in the treatment of tuberculosis using resorbable oral doses ranging from 8 to $12 \mathrm{~g} / \mathrm{d} .{ }^{37}$ With this indication profound experience with side effects of the drug has been gathered in a large number of patients. The most common side effects include nausea, vomiting, and epigastric pain. In a small number of cases fever, joint pains, skin eruptions, and hepatitis have been reported, all of which may be attributed to hypersensitivity reactions. ${ }^{38}$ Very rarely seen are more serious side effects such as leucopenia, thrombo- 
cytopenia, and agranulocytosis. ${ }^{34} 3738$ We therefore speculate that 4-ASA may be used at much higher doses as a slow release formulation and might have a much higher therapeutic efficacy in IBD than seen in this and previous studies.

We concluded that oral 4-ASA treatment may be as effective as 5-ASA in the maintenance of remission in Crohn's disease. Moreover, several studies have suggested that 4-ASA may be effective in the treatment of active Crohn's disease or ulcerative colitis both as a topical formulation (enema) or as an oral slow release tablet. Further placebo controlled trials including a large number of patients are warranted to further investigate this promising compound.

We greatly appreciate the help of S Hoyer and B Kaiser in the conduction of the study and the critical discussion of the results by $\mathrm{D}$ Berghaus and $\mathrm{H} C$ Reinecker

Parts of this work have been presented at the 93rd annual meeting of the American Gastroenterological Association, San Francisco, CA, USA (1992) and at a Falk meeting in Quebec City, Quebec, Canada (1992). This work was supported by a grant from Block Drug Co (to AR).

1 Summers RW, Switz DM, Sessions JT Jr, Becktel JM, Best WR, Kern J Jr, et al. National Cooperative Crohn's Disease Study: results of drug treatment. Gastroenterology 1979; 77: 847-69.

2 Malchow H, Ewe K, Brandes JW, Goebell H, Ehms H, Somer H, et al. European Cooperative Crohn's Disease Study: results of drug treatment. Gastroenterology 1984; 86: $249-66$.

3 Thompson ABR. New developments in the use of 5 -aminosalicylic acid in patients with inflammatory bowel disease. Aliment Pharmacol Therap 1991; 5: 449-70.

4 Singleton JW, Hanauer SB, Gitnick GL, Peppercorn MA, Robinson MG, Wruble LD, et al. Mesalamine capsules for the treatment of active Crohn's disease: results of a 16-week trial. Pentasa Crohn's Disease Study Group. Gastroenterology 1993; 104: 1293-301.

5 Campieri M, Gionchetti P, Beluzzi A, Brignola C, Migaldi $M$, Tabanelli GM, et al. Efficacy of 5-ASA enemas versus hydrocortisone enemas in ulcerative colitis. Dig Dis Sci 1987; 32: 67-70S.

6 Rachmilewitz D, with International Study Group. Coated mesalazine (5-aminosalicylic acid) versus sulphasalazine in the treatment of active ulcerative colitis: a randomised trial. BMF 1989; 298: 82-6.

7 Rutgeerts $P$, with International Study Group. Comparative efficacy of coated, oral 5-aminosalicylic acid (Claversal) and sulphasalazine for maintaining remission of ulcerative colitis. Aliment Pharmacol Ther 1989; 3: 183-91.

8 Miner P, Schwartz J, Aora S. Maintenance of remission in ulcerative colitis (UC) patients with controlled-release mesalamine capsules (Pentasa), Gastroenterology 1992; 102: A666.

9 Brignola C, Ianonne $M$, Pasquali S, Campieri $M$, Gionchetti P, Beluzzi A, et al. Placebo controlled trial of oral 5-ASA in relapse prevention of Crohn's disease. oral 5-ASA in relapse pre

10 Bondesen S and the Danish 5-ASA Group. Mesalazine (Pentasa) as prophylaxis in Crohn's disease. A multicentre, controlled trial. Scand $\mathcal{F}$ Gastroenterol 1991; 26 (suppl 183): F44.

11 Prantera C, Pallone F, Brunetti G, Cottone M, Miglioli M and the Italian IBD study group. Oral 5-aminosalicylic acid (Asacol) in the maintenance treatment of Crohn's disease. Gastroenterology 1992; 103: 363-8.

12 Gendre JP, Mary JY, Florent C, Modigliani R, Coombel JF, Soule JC, et al. Oral mesalazine (Pentasa) as maintenance treatment in Crohn's disease: a multicentre, placebocontrolled study. Gastroenterology 1993; 104: 435-9.

13 Thompson ABR and the International Mesalamine Study Group. Coated oral 5-aminosalicylic acid versus placebo in maintaining remission in inactive Crohn's disease. in maintaining remission in inactive

14 Lover ML. United States patent no 4440763 , April 3, 1984.

15 Campieri M, Lanfranchi GA, Bertoni F, Brignola C,
Bazzochi G, Minguzzi MR, et al. A double blind clinical trial to compare the effect of 4-aminosalicylic acid in topical treatment of ulcerative colitis. Digestion 1984; 29: topical

16 Gandolfo J, Farthing M, Powers G, Eagen K, Goldberg M, Berman P, et al. 4-Aminosalicylic acid retention enemas in the treatment of distal colitis. Dig Dis Sci 1987; 32: 700-4.

17 Ginsberg AL, Beck LS, McIntosh TM, Nochomovitz LE. Treatment of left-sided ulcerative colitis with 4-aminosalicylic acid enemas: a double blind, placebo-controlled trial. Ann Intern Med 1988; 108: 195-9.

18 Nagy F, Karacsony G, Varro V. Experience with topical administration of 4-aminosalicylic acid in ulcerative colitis. Dis Colon Rectum 1989; 3: 134-7.

19 Selby WS, Bennett MK, Jewell DP. Topical treatment of distal ulcerative colitis with 4-aminosalicylic acid enemas. Digestion 1984; 29: 231-4

20 O'Donnell LJD, Arvind AS, Hoang P, Cameron D, Talbot JC, Jewell DP, et al. Double blind, controlled trial of 4 -aminosalicylic acid and prednisolone enemas in distal ulcerative colitis. Gut 1992; 33: 947-9.

21 Ginsberg L, Davis ND, Nochomovitz LE. Placebocontrolled trial of ulcerative colitis with oral 4-aminosalicylic acid. Gastroenterology 1992; 102: 448-52.

22 Schreiber S, MacDermott RP, Raedler A, Pinnau R, Bertovich M, Nash GS. Increased activation of intestinal lamina propria mononuclear cells in inflammatory bowel disease. Gastroenterology 1991; 101: 1020-30.

23 Schreiber S, Raedler A, Conn AF, Rombeau JL, MacDermott RP. Increased release of soluble interleukin-2 receptor by colonic lamina propria mononuclear cells in inflammatory bowel disease. Gut 1992; 32: cells in

24 Raedler A, Fraenkel S, Klose G, Thiele HG. Involvement of the immune system in the pathogenesis of Crohn's disease. Expression of the T9 antigen on peripheral immunocytes correlates with the severity of the disease. Gastroenterology 1985; 88: 978-83.

25 Mueller CH, Knoflach P, Zielinski CC. T cell activation in Crohn's disease. Intestinal levels of soluble interleukin-2 receptor in serum and in supernatants of stimulated peripheral blood mononuclear cells. Gastroenterology 1990; 98: 639-46.

26 Crabtree JE, Juby LD, Heatly RV, Lobo AJ, Bullimore DW, Axon ATR. Soluble interleukin-2 receptor in Crohn's disease: relation of serum concentrations to disease activity. Gut 1990; 31: 1033-6.

27 Surmann R, Surmann M, Schneider M, Gerlach U. Soluble interleukin-2 receptor as an indicator of disease activity in patients with ulcerative colitis. Presented at the Falk Symposium: inflammatory bowel diseases (abstract 70), Freiburg, Germany, October 18-20, 1990

28 Hawkey CJ, Truelove SC. Inhibition of prostaglandin synthetase in human rectal mucosa. Gut 1983; 24: 213-7.

29 Hawkey CJ, Boughton-Smith NK, Whittle BJR. Modulation of human colonic arachidonic acid metabolism by sulphasalazine. Dig Dis Sci 1985; 130: 1161-5.

30 Sharon P, Stenson WF. Enhanced synthesis of leukotriene B4 by colonic mucosa in inflammatory bowel disease. Gastroenterology 1984; 86: 453-60.

31 Ahnfelt-Ronne I, Nielsen OH, Christensen A, Langholz E, Binder V, Riis P. Clinical evidence supporting the radical scavenger mechanism of 5-ASA. Gastroenterology 1990; 98: $1162-9$.

32 Miyachi Y, Yoshioka A, Imamura S, Niwa Y. Effect of sulphasalazine and its metabolites on the generation of reactive oxygen species. Gut 1987; 28: 190-5.

33 Williams JG, Hallett MB. Effect of sulphasalazine and its active metabolite, 5-aminosalicylic acid, on toxic oxygen metabolite production by neutrophils. Gut 1989; 30: 1581-7.

34 Nielsen OH, Ahnfelt-Ronne I. 4-Aminosalicylic acid has no effect on arachidonic acid metabolism in human neutrophils or on the free radical, $1 \cdot 1$ diphenyl-2-picrylhydrazyl. Pharmacol Toxicol 1988; 62: 223-6.

35 Schreiber S, Raedler A, Stenson WF, MacDermott RP. The immunology of inflammatory bowel disease. Gastroenterol Clin North Am 1992; 21: 451-502.

36 von Ritter C, Grisham MB, Granger DN. Sulfasalazine metabolites and dapsone attenuate formyl-methionylleucyl-phenylalanine-induced mucosal injury in rat ileum Gastroenterology 1989; 96: 811-6.

37 Schless JM, Inglis RM, Hammond JH, Hale JM. Comparative evaluation of blood levels and tolerance of sodium PAS and conjugated PAS and ascorbic acid. Dis Chest 1966; 50: 595-600.

38 Cannemeyer W, Thompson JR, Lichtenstein MR. Severe paraaminosalicylic acid hypersensitivity: blood and lymph node studies. Blood 1955; 10: 62-75. 